\title{
BMJ Open Acupuncture at Houxi (SI 3) acupoint for acute neck pain caused by stiff neck: study protocol for a pilot randomised controlled trial
}

\author{
Zhong-ren Sun, ${ }^{1,2}$ Jin-huan Yue, ${ }^{1}$ Hong-zhao Tian, ${ }^{1}$ Qin-hong Zhang ${ }^{1,3}$
}

To cite: Sun Z-ren, Yue Jhuan, Tian $\mathrm{H}-z h a 0$, et al. Acupuncture at Houxi (SI 3) acupoint for acute neck pain caused by stiff neck: study protocol for a pilot randomised controlled trial. BMJ Open 2014;4:e006236. doi:10.1136/bmjopen-2014006236

- Prepublication history for this paper is available online. To view these files please visit the journal online (http://dx.doi.org/10.1136/ bmjopen-2014-006236).

Received 29 July 2014 Accepted 19 August 2014

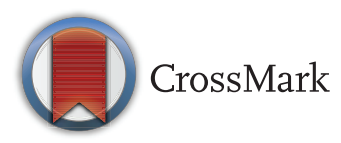

${ }^{1}$ Department of Acupuncture and Moxibustion, College of Acupuncture and

Moxibustion, Heilongjiang University of Chinese Medicine, Harbin, China ${ }^{2}$ Department of Acupuncture and Moxibustion, First Affiliated Hospital of Heilongjiang University of Chinese Medicine, Harbin, China

${ }^{3}$ Department of Acupuncture and Moxibustion, Second Affiliated Hospital of Heilongjiang University of Chinese Medicine, Harbin, China

Correspondence to Dr Qin-hong Zhang; zhangqh0451@163.com

\section{ABSTRACT}

Introduction: The use of acupuncture has been suggested for the treatment of acute neck pain caused by stiff neck in China. However, current evidence is insufficient to draw any conclusions about its efficacy. Therefore this pilot study was designed to evaluate the feasibility and efficacy of acupuncture at the Houxi $(S / 3)$ acupoint for treatment of acute neck pain.

Methods/analysis: This pilot study will be a twoparallel-group, assessor-blinded, randomised controlled trial. Thirty-six stiff neck participants with acute neck pain will be recruited and randomly divided into two groups in a 1:1 ratio. Participants in the control group will receive massage on the local neck region (5 min each session, three times a day for 3 days). In addition to massage, patients in the treatment group will receive acupuncture (one session a day for 3 days). Measures will be taken at 0,3 and 15 days. The primary outcome is the Northwick Park Neck Pain Questionnaire (NPQ). The secondary outcome is the Short Form of the McGill Pain Questionnaire (SF-MPQ).

Ethics/dissemination: The protocol for this pilot randomised clinical trial has undergone ethics scrutiny and been approved by the ethics review boards of the First Affiliated Hospital of Heilongjiang University of Traditional Chinese Medicine (Permission number: HZYLL201303502). The findings of this study will provide important clinical evidence on the feasibility and efficacy of acupuncture treatment for stiff neck patients with acute neck pain. In addition, it will explore the feasibility of further acupuncture research.

Trial registration number: ChiCTR-TRC-13003911.

\section{BACKGROUND}

Stiff neck (also known as 'Laozhen' in China) is commonly seen in clinics. It is often caused by unsuitable sleep posture. Its main symptom is the sharp onset of severe and persistent pain in the local cervical region. ${ }^{1}$ It presents rigidly in the erect central position and resists the least movement, active and passive, in any direction. ${ }^{1}$ The pain is greatly augmented by such attempts.
The current treatments for stiff neck patients with acute neck pain include nerve blocking therapy, ${ }^{2}$ Chinese herbal medicine decoction, ${ }^{3}$ moxibustion, ${ }^{4}$ tuina,${ }^{5}$ massage,${ }^{6}$ cupping therapy, ${ }^{7}$ Gua Sha therapy ${ }^{8}$ and acupressure. ${ }^{9}$ However, most of these treatments are limited by their modest effectiveness.

Acupuncture therapy has been widely used to treat a variety of conditions, such as allergic rhinitis, ${ }^{10}$ stroke, ${ }^{11}$ insomnia, ${ }^{12}$ migraine,,${ }^{13}$ low back pain, ${ }^{14}$ frozen shoulder, ${ }^{15}$ pressure ulcer $^{16}$ and neck pain ${ }^{17}$. In addition, the evidenced-based potential for effective use of acupuncture therapy for stiff neck patients with acute neck pain has been suggested in China. ${ }^{18}{ }^{19}$ However, currently, the evidence is poor because of methodological limitations, such as low-quality study design, small sample size, inadequate control groups, no follow-up, lack of suitable outcome measures, and variation in acupuncture composition. Therefore, more rigorously designed studies are needed to elucidate the feasibility and effectiveness of acupuncture for stiff neck patients with acute neck pain.

In this study, we aimed to conduct a randomised controlled trial (RCT) in stiff neck patients with acute neck pain to assess the feasibility and efficacy of acupuncture at the Houxi (SI 3) acupoint (on the dorsum of the hand, in the depression proximal to the ulnar side of the fifth metacarpophalangeal joint, at the border between the red and white flesh) (figure 1) compared with massage intervention. The Northwick Park Neck Pain Questionnaire (NPQ) and the Short Form of the McGill Pain Questionnaire (SF-MPQ) will be used as the primary and secondary outcomes, respectively. The results of this study will be used to explore the feasibility and efficacy of acupuncture therapy and also provide the data to calculate the appropriate sample size for future studies. 


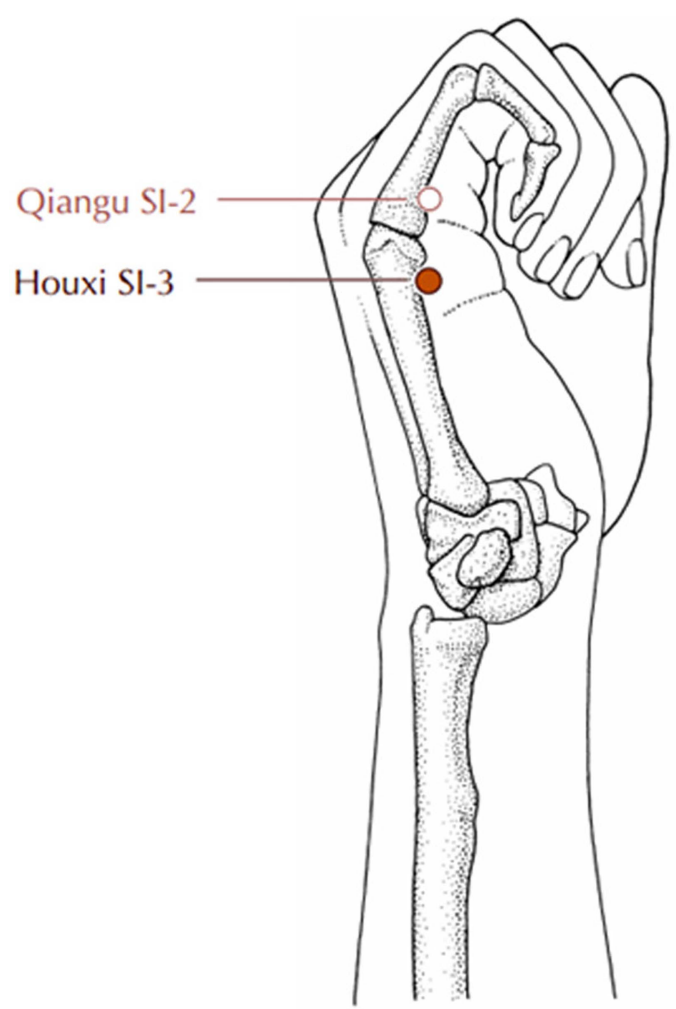

Figure 1 Location of Houxi (SI 3).

\section{METHODS/DESIGN Objective}

The aim of this study was to assess the feasibility and efficacy of acupuncture therapy for stiff neck patients with acute neck pain.

\section{Design}

This pilot study is to be a two-parallel-group, assessorblinded RCT. It will be conducted at the clinical research centre of the First Affiliated Hospital of Heilongjiang University of Chinese Medicine. The protocol has been registered with the Chinese Clinical Trial Registry, a national public clinical trial registry and a recognised primary registry of the WHO. Eligible subjects will be randomly divided into two groups (acupuncture group and control group) in a 1:1 allocation ratio and will receive the intervention (one session daily for 3 days, with 15 days follow-up) (figure 2, table 1). The outcome evaluation and data analysis will be conducted by professionals who are blinded to the group allocation.

\section{Eligibility}

\section{Inclusion criteria}

- Age 18-65 years old (both male and female participants)

- A confirmed diagnosis of acute neck pain caused by stiff neck according to the diagnostic criteria and referring to the Diagnostic efficacy of standard traditional Chinese medicine syndrome. ${ }^{20}$

- No acupuncture intervention or other related therapy in the 3 days before study entry.

- Informed consent document has been signed. ${ }^{17}$

\section{Exclusion criteria}

- History of neck trauma, cervical fracture or cervical surgery, or spinal canal cancer. ${ }^{17}$

- Acute neck pain with ulcers on the neck region.

- Complications of severe systemic diseases, such as cardiocerebrovascular disease, diabetes mellitus, kidney disease or digestive system disease. ${ }^{17}$

- Rejection or fear of acupuncture therapy. ${ }^{17}$

- Any type of acupuncture intervention used in the 7 days before study entry.

- Failure to accept completion of clinical treatment.

\section{Randomisation and allocation concealment}

Subjects who meet the inclusion criteria will be randomly assigned to the acupuncture group or the control group in a 1:1 ratio at the second visit through central
Figure 2 Flow chart of study process. NPQ, Northwick Park Neck Pain Questionnaire; SF-MPQ, Short Form of McGill Pain Questionnaire.

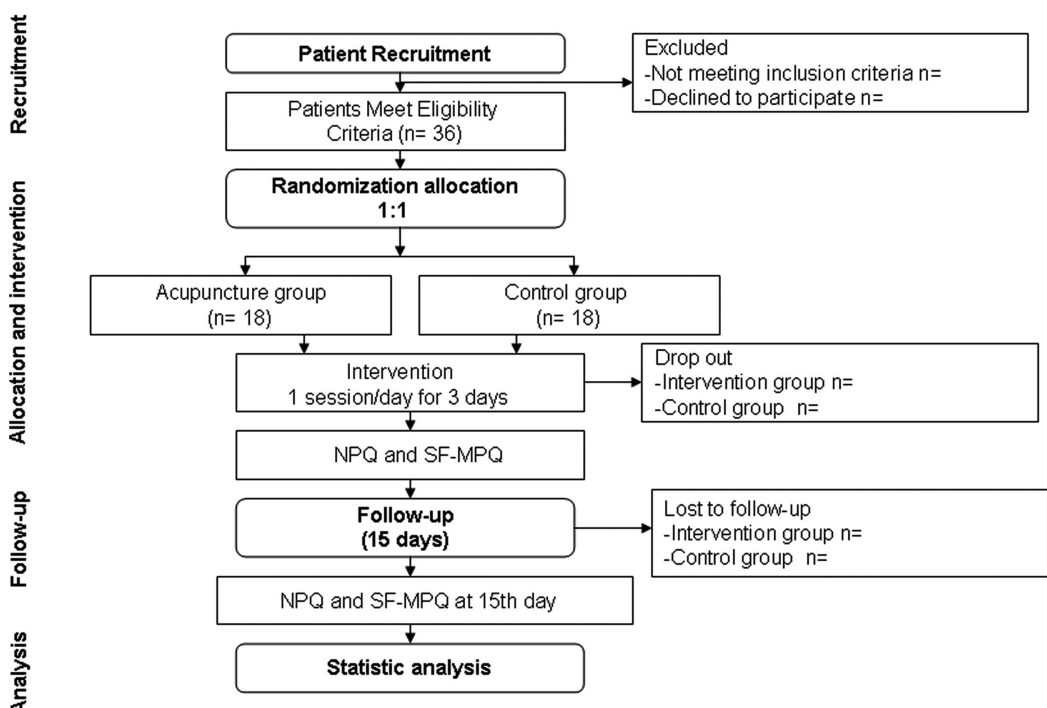


Table 1 Time to visit and data collection

\begin{tabular}{|c|c|c|c|c|}
\hline & $\begin{array}{l}-1 \text { day } \\
\text { Baseline }\end{array}$ & $\begin{array}{l}0 \text { day } \\
\text { Treatment phase }\end{array}$ & 3 days & $\begin{array}{l}15 \text { days } \\
\text { Follow-up phase } \\
\text { (end of treatment) }\end{array}$ \\
\hline \multicolumn{5}{|l|}{ Patients } \\
\hline Informed consent & $x$ & & & \\
\hline Sign informed consent & & $x$ & & \\
\hline Medical history & $x$ & & & \\
\hline Physical examination & $x$ & & & \\
\hline Randomisation & & $x$ & & \\
\hline \multicolumn{5}{|l|}{ Intervention } \\
\hline Acupuncture group $(n=18)$ & & $\begin{array}{l}3 \text { sessions of acup } \\
\text { (SI 3) acupoint plu }\end{array}$ & thouxi & \\
\hline \multicolumn{5}{|l|}{ Comparisons } \\
\hline Control group $(n=18)$ & & $\begin{array}{l}3 \text { sessions of mas } \\
\text { neck region }\end{array}$ & cal & \\
\hline \multicolumn{5}{|l|}{ Outcomes } \\
\hline NPQ & & $\times$ & $\times$ & $x$ \\
\hline SF-MPQ & & $x$ & $x$ & $x$ \\
\hline \multicolumn{5}{|l|}{ Participant safety } \\
\hline Adverse events & & $x$ & $x$ & $x$ \\
\hline
\end{tabular}

randomisation. Random numbers will be generated using a computerised number generator with the SAS v8.1 software package by a professional statistician, who will be blinded to the study. Acupuncturists not involved in the process of randomised assignment will then deliver the interventions.

Allocation concealment will be achieved by using sequentially numbered opaque sealed envelopes containing the randomised assignments. The envelopes will be opened only after the subjects have met all selected criteria and finished the baseline evaluation. To our knowledge, it is not practical to blind the acupuncturists to intervention allocations and patient symptoms. The participants will also know the allocated group. However, the outcome assessors and data analysts will be blinded to the treatment allocation. ${ }^{21}$ Patients will be recruited through the clinic of the Clinical Medicine Research Center of the First Affiliated Hospital of Heilongjiang University of Chinese Medicine.

\section{Intervention}

\section{Control group}

The following massage therapy will be given to the control group: grasp the neck with one hand, putting the thumb on one side of the neck and the fingers on the other side; knead the neck on both sides by digging in the thumb and fingers and gently squeezing them together; repeat the motion up and down the neck for 5 min each time, three times daily for 3 days.

\section{Acupuncture group}

In addition to the massage treatment, acupuncture therapy will be used in this group. Subjects will receive acupuncture at the Houxi (SI 3) acupoint on the painful side of the neck. Sterile disposable acupuncture needles (40 $\mathrm{mm}$ in length and $0.30 \mathrm{~mm}$ in diameter; Andy brand, Guizhou Andy Medical Instrument Co) will be perpendicularly operated to a depth of $10-15 \mathrm{~mm}$ on the Houxi (SI 3) acupoint. ${ }^{17}$ Acupuncture will be administered for 30 min each session, with one session a day for 3 days.

\section{Outcomes}

Two well-recognised patient-reported tools will be used to measure the outcomes. ${ }^{17} \mathrm{NPQ}$ will be used as the primary outcome measure, and SF-MPQ as the secondary outcome measure.

\section{Primary outcome measurement}

The NPQ tool will be used to evaluate acute neck pain. ${ }^{22}$ A higher score reflects a more serious disease state.

\section{Secondary outcome measurement}

The SF-MPQ tool will be used to measure neck pain intensity. A higher SF-MPQ score reflects more serious pain. ${ }^{23}$ This tool comprises three parts: the main component (comprising 15 descriptors (11 sensory; 4 affective)); the visual analogue scale $(10 \mathrm{~cm}$ horizontal line with clearly defined boundaries, which ranges from 'no pain' to the 'worst possible pain'); and the Present Pain Intensity index (six-point verbal rating scale).

\section{Sample size}

This pilot study aims to assess the feasibility of acupuncture for stiff neck patients with acute neck pain, and also the feasibility of a further large clinical trial. ${ }^{17}$ Because of its short duration (3 days), the desired and optimised sample size for this pilot trial is 36 subjects, 18 in each group. It assumes a $20 \%$ drop-out rate and a 
minimum sample size sufficient to assess the feasibility and efficacy of acupuncture. ${ }^{24}$

\section{Statistical analysis}

All analyses will be conducted by a statistician blinded to the group allocation using the SPSS v17.0 and SAS v8.1 software packages. Significance levels will be reported at $\mathrm{p}<0.05$. The intention-to-treat principle will be used to analyse the data of baseline characteristics and primary and secondary outcomes. If an adjustment for possible baseline incomparability is needed, covariance analysis will be performed. If the measurement data from NPQ and SF-MPQ have non-normal distribution, the Wilcoxon rank sum test will be used. If the measurement data have normal distribution, the $t$ test will be used.

\section{Adverse events}

Any adverse events (expected or unexpected) related to acupuncture therapy will be reported to the investigator or physicians by participants and recorded by the investigator at every visit. Possible adverse events related to acupuncture are local bleeding or oedema resulting from bleeding, redness, itching, and dizziness or fainting, throughout treatment and the follow-up period. ${ }^{25}$ Subjects will receive appropriate intervention if any adverse events occur. Serious adverse events will be reported to the primary investigator immediately, and participants will be withdrawn from the study. The following details on adverse events will be documented: date of occurrence; time lost; measures taken related to the acupuncture; causal relationship to acupuncture; treatment for adverse event.

\section{Ethics}

The protocol of this pilot study has been peer reviewed and approved by the ethics review boards of the First Affiliated Hospital of Heilongjiang University of Traditional Chinese Medicine (Permission number: HZYLL201303502). Written informed consent will be acquired from all study subjects before enrolment into the study.

\section{DISCUSSION}

Acute neck pain caused by stiff neck is a common public health problem that presents to the clinic. The results of this pilot study will focus on stiff neck patients with acute neck pain and will establish if acupuncture is a feasible and effective therapy for relief of this kind of clinical manifestation.

The Houxi (SI 3) acupoint is the Shu point of five Shu points (special points of the 12 meridians, located distal to the elbows and knees, namely well, brook, stream, river and sea points), which belongs to the small intestine channel. According to the theory of Five-Shu points, the Shu point (SI 3) is responsible for diseases related to neck pain, especially acute neck pain caused by stiff neck. ${ }^{25}$

The outcomes of NPQ and SF-MPQ are well established for evaluating the acute neck pain of stiff neck. This pilot study will provide data on the feasibility and efficacy of acupuncture therapy for relieving neck pain and decreasing intervention duration throughout a follow-up period of 15 days after treatment completion. The clinical outcome evaluation with NPQ and SF-MPQ will be used in further long-term clinical studies.

Besides the appropriate outcome measures, the proper use of a control group is also a critical issue in designing a high-quality clinical trial. The aim of this trial is to illustrate whether acupuncture is feasible and effective for reducing neck pain. In addition, a pragmatic design using massage intervention in the control group was planned. This trial using comparison of acupuncture plus massage with massage only should provide evidence on the feasibility and efficacy of acupuncture.

In conclusion, this pilot RCT aims to evaluate the feasibility and effectiveness of acupuncture for stiff neck patients with acute neck pain, as well as its feasibility and efficacy in future episodes and in further large clinical studies. Its results will shed new light on acupuncture therapy for acute neck pain.

Acknowledgements This work was supported in part by the Outstanding Innovative Talents Supporting Project of Heilongjiang University of Chinese Medicine (grant number 2012RCL01; 2012RCQ64).

Contributors J-hY and Q-hZ contributed equally to this work. Q-hZ conceived the study and designed the study protocol. J-hY and $\mathrm{H}-\mathrm{zT}$ drafted the manuscript. Z-rS sought funding and ethics approval. All authors read and approved the final manuscript.

Competing interests None.

Patient consent Obtained.

Ethics approval First Affiliated Hospital of Heilongjiang University of Chinese Medicine.

Provenance and peer review Not commissioned; peer reviewed for ethics and funding approval prior to submission.

Open Access This is an Open Access article distributed in accordance with the Creative Commons Attribution Non Commercial (CC BY-NC 4.0) license, which permits others to distribute, remix, adapt, build upon this work noncommercially, and license their derivative works on different terms, provided the original work is properly cited and the use is non-commercial. See: http:// creativecommons.org/licenses/by-nc/4.0/

\section{REFERENCES}

1. Shafar J. Stiff-neck syndrome. Br Med J 1978;1:511.

2. Pan T. Nerve blocking therapy cervical plexus nerve for stiff neck. Chin J Orthop 1999;12:36.

3. Zhang HC. Clinical observation of $\mathrm{Ge}$ gen decoction for stiff neck with 38 cases. Nei Mongol J Tradit Chin Med 2010;2:125-6.

4. Xu FR. Warm ginger moxibustion on Jingqu ( $L$ 8) point for stiff neck. Chin Acupunct Moxibustion 2008;28:652.

5. Wang $X Y$. Clinical observation on 50 cases of strained neck treated with tuina manipulations. J Beijing Univ Tradit Chin Med (Clin Med) 2010;17:12-13.

6. Yao CP. Massage therapy for stiff neck. Sichuan J Tradit Chin Med 1984;2:41.

7. Jiang SK. Cupping therapy for stiff neck with 15 cases. J Clin Med Offic 2002;30:8.

8. Gu LX. Gua Sha therapy for stiff neck with 20 cases. Shanghai J Acupunct Moxibustion 1995;14:263.

9. Liu YL, Ma YC, Li H. Clinical experience of acupressure therapy for stiff neck with 56 cases. J Changchun Univ Tradit Chin Med 2008;24:720.

10. Xue CC, English R, Zhang JJ, et al. Effect of acupuncture in the treatment of seasonal allergic rhinitis: a randomized controlled clinical trial. Am J Chin Med 2002;30:1-11.

11. Gosman-Hedström G, Claesson L, Klingenstierna U, et al. Effects of acupuncture treatment on daily life activities and quality of life: 
a controlled, prospective, and randomized study of acute stroke patients. Stroke 1998;29:2100-8.

12. Cheuk DKL, Yeung WF, Chung KF, et al. Acupuncture for insomnia. Cochrane Database Syst Rev 2007;(3):CD005472.

13. Diener HC, Kronfeld K, Boewing G, et al; GERAC Migraine Study Group. Efficacy of acupuncture for the prophylaxis of migraine: a multicentre randomised controlled clinical trial. Lancet Neurol 2006;5:310-16.

14. Furlan AD, van Tulder MW, Cherkin DC, et al. Acupuncture and dry-needling for low back pain. Cochrane Database Syst Rev 2005; (1):CD001351.

15. Sun KO, Chan KC, Lo SL, et al. Acupuncture for frozen shoulder. Hong Kong Med J 2001;7:381-91.

16. Yue J, Zhang Q, Sun Z, et al. A case of electroacupuncture therapy for pressure ulcer. Acupunct Med 2013;31:450-1.

17. Sun ZR, Yue JH, Zhang QH. Electroacupuncture at Jing-jiaji points for neck pain caused by cervical spondylosis: a study protocol for a randomized controlled pilot trial. Trials 2013;14:360.
18. Wu YC, Zhang JF, Wang CM, et al. Clinical study of Tuina for stiff neck. J Acupunct Tuina Sci 2009;7:225-7.

19. Hu SG, Zhong YQ. Clinical observations on the treatment of 50 cases of stiff neck by acupuncture. J Tradit Chin Med 1992;12:57-8.

20. State Administration of Traditional Chinese Medicine. Diagnostic efficacy of standard Traditional Chinese Medicine Syndrome. Nanjing: Press of Nanjing University, 1994.

21. Altman DG, Dore CJ. Randomisation and baseline comparisons in clinical trials. Lancet 1990;335:149-53.

22. Leak AM, Cooper J, Dyer S, et al. The Northwick Park neck pain questionnaire, devised to measure neck pain and disability. $\mathrm{Br} J$ Rheumatol 1994;33:469-74.

23. Melzack R. The short-form McGill Pain Questionnaire. Pain 1987;30:191-7.

24. Johanson GA, Brooks GP. Initial scale development: sample size for pilot studies. Educ Psychol Meas 2010;70:394-400.

25. Shi XM. Acupuncture and Moxibustion. 1st edn. Beijing: China Press of Traditional Chinese Medicine, 2002. 